\title{
Reconciling Exhaustive Pattern Matching with Objects
}

\author{
Chinawat Isradisaikul Andrew C. Myers \\ Department of Computer Science \\ Cornell University \\ Ithaca, New York, United States \\ chinawat@cs.cornell.edu_andru@cs.cornell.edu
}

\begin{abstract}
Pattern matching, an important feature of functional languages, is in conflict with data abstraction and extensibility, which are central to object-oriented languages. Modal abstraction offers an integration of deep pattern matching and convenient iteration abstractions into an object-oriented setting; however, because of data abstraction, it is challenging for a compiler to statically verify properties such as exhaustiveness. In this work, we extend modal abstraction in the JMatch language to support static, modular reasoning about exhaustiveness and redundancy. New matching specifications allow these properties to be checked using an SMT solver. We also introduce expressive pattern-matching constructs. Our evaluation shows that these new features enable more concise code and that the performance of checking exhaustiveness and redundancy is acceptable.
\end{abstract}

Categories and Subject Descriptors D.3.3 [Programming Languages]: Language Constructs and Features-Patterns, Polymorphism; D.1.5 [Programming Techniques]: Object-oriented Programming; F.3.1 [Logics and Meanings of Programs]: Specifying and Verifying and Reasoning about Programs-Pre- and postconditions, Mechanical verification

General Terms Design, Verification, Languages

Keywords JMatch; pattern matching; named constructor; equality constructor; matching specification; exhaustiveness; redundancy; modal abstraction; Java; data abstraction; subtyping

\section{Introduction}

Despite being an important feature of modern functional programming languages, pattern matching has not been adopted by most object-oriented languages. Data abstraction and extensibility, both primary goals of object-oriented languages, conflict with pattern matching. This work explores a language design for integrating pattern matching with object-oriented programming.

The following is a simple implementation of natural numbers in OCaml. The algebraic data type nat, with two constructors Zero and Succ, represents a natural number; the recursive plus function adds two naturals by matching them with one of three patterns.

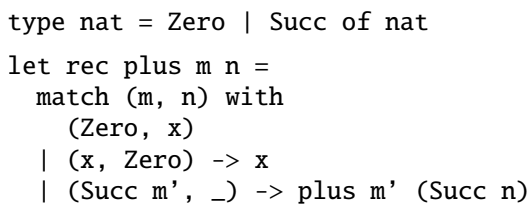

Copyright (C) ACM, 2013. This is the author's version of the work. It is posted here by permission of ACM for your personal use. Not for redistribution.

PLDI'13 Seattle, Washington, USA.

Copyright (C) 2013 ACM 978-1-4503-2014-6/13/06...\$15.00
This example illustrates two benefits of pattern matching in ML and other functional programming languages such as Haskell.

The first benefit is that patterns serve a dual role that enables algebraic reasoning and results in concise, intuitive code. A constructor such as Succ is also a pattern that matches the values produced by that constructor. Patterns can be nested to match complex values in a natural way, so a pattern like Succ(Succ (n)) matches exactly the values constructed by expressions using the same syntax.

The second benefit is that pattern matching helps catch common programming errors. Patterns in a match expression can be checked to ensure that they are exhaustive and not redundant: that all possible values are matched by some pattern, and that every pattern can match some value. Without such checks, if the programmer forgot the first of the three cases above, the program could crash with an exception. With such checks, the compiler would warn that no cases match values of the form (Zero, Succ _).

Relying on access to the concrete representation of data, however, makes the ML-style pattern matching inimical to data abstraction [30]. A value produced by one module can only be matched by patterns in another module if the second module knows the underlying representation of the value. Agreement on the concrete representation tightly couples the two modules in a way usually considered undesirable for large software systems. For example, we might initially implement natural numbers as above, then later want to change the representation to be an int. This change is not possible in ML without breaking client code.

To make pattern matching compatible with data abstraction, prior work has developed pattern-matching constructs that can be implemented by arbitrary code. Examples of this approach include views [30], extractors [5], and active patterns [29]. These mechanisms permit matching on deep patterns over abstract data, but sacrifice other benefits of algebraic pattern matching. There is no check that patterns are consistent with their corresponding constructors, so algebraic reasoning is weakened. Further, data abstraction interferes with checking exhaustiveness and redundancy.

The JMatch language [19] introduced another way to harmoniously integrate pattern matching into object-oriented languages, through modal abstractions that support multiple directions of computation. Modal abstractions allow a constructor and its pattern to be implemented by the same invertible computation, ensuring that they are inverses. Determining whether patterns are exhaustive or redundant, however, remained impossible under the data abstraction provided by JMatch. Furthermore, the added expressive power of patterns implemented by complex computations means that programmers can accidentally omit patterns more easily than with algebraic data types.

The challenge for analysis of exhaustiveness and redundancy is to reason statically without violating data abstraction. The main contribution of this paper is, therefore, a way to extend modal abstractions with concise specifications that enable static reasoning 


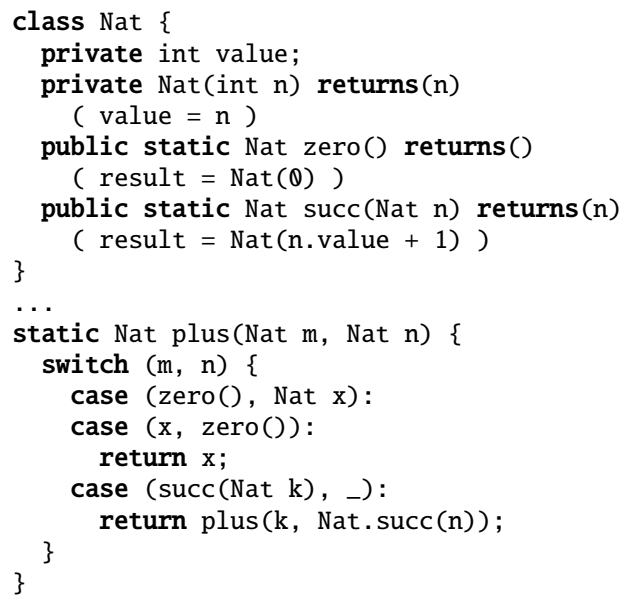

Figure 1. Natural numbers with data abstraction in JMatch.

about exhaustiveness and redundancy of pattern matching and, more generally, about the totality of computations.

Object-oriented programming involves more than just data abstraction; subtyping and inheritance are key ingredients supporting extensibility. For extensibility, different implementations of (subtypes of) the same interface should support the same patterns without clients knowing which implementation has been used. We therefore introduce named constructors that can be used as patterns in this way. We also introduce two first-class or-patterns that generalize both data-type constructors and or-patterns in ML.

We proceed as follows. Section 2 reviews modal abstraction in JMatch. Section 3 introduces mechanisms that improve the expressive power of pattern matching and its integration with objects. Section 4 describes new static annotations that support reasoning about exhaustiveness and redundancy. The verification procedure is explained in Section 5. Section 6 describes our implementation of an extended version of JMatch. Using various code examples, we evaluate its expressiveness, analytic power, and efficiency in Section 7. Section 8 discusses related work, and Section 9 concludes.

\section{Background}

Some background will be helpful on JMatch [17, 19], an extension to Java 1.4 that supports pattern matching and iteration through modal abstraction.

\subsection{Modal abstraction}

Section 1 observed that in OCaml, natural numbers cannot support pattern matching while being represented internally with an int. Figure 1 shows how this can be done in JMatch. The key idea is that JMatch methods may declare multiple modes that correspond to different "directions" of evaluation, analogously to predicate mode declarations in the logic programming language Mercury [26]. In addition to the ordinary forward mode, which acts like a Java method, a JMatch method may also provide backward modes which, given a desired result, compute corresponding argument values. Backward modes support pattern matching. For example, the method succ may be used in the forward mode to compute the successor of a number. As indicated by the clause returns (n) on line 7 , it also has a backward mode that computes the number $n$ for which the value given in result is the successor.

This implementation of Nat is more complex than in OCaml because the abstract view that supports pattern matching must be related to its concrete representation as an int. The methods of
Nat demonstrates that JMatch programs can define patterns that both preserve data abstraction, because the field value is private, and are also usable outside the module that defines them. Lines 1119 show how the backward modes of these methods can be used to implement the method plus similarly to the earlier OCaml code.

In general, a JMatch method implements a relation over its arguments and its result. Each of its modes is a different way of exploring the relation. For example, the succ operation is a binary relation on Nat: a subset of Nat $\times$ Nat. In each mode, some of the arguments or the return value are knowns supplied by the caller, and the others are unknowns to be solved for.

Individual method modes may be implemented by imperative, Java-like code, but one single declarative-style implementation of multiple modes is often more concise. As in each of Nat's methods in Figure 1, a declarative method implementation is a boolean formula placed inside parentheses, directly expressing the implemented relation. For example, the equation result $=$ $\operatorname{Nat}(n$.value +1$)$ at line 8 exactly captures the succ relation. ${ }^{1}$ For each mode of a method, the compiler generates an imperative algorithm that, given values for knowns, finds values of all unknowns that satisfy the formula. Thus, the backward mode often comes nearly for free, unlike with related approaches such as extractors [5].

Not only user-defined abstractions but also built-in types such as primitive types support modal abstractions. For example, integer operations such as + and - can solve for either of their arguments, given a result to match against.

\subsection{Iterative modes}

Modes need not be functions; viewed as relations, they may be one-to-many or many-to-many. A mode is iterative when there may be more than one solution to the unknowns for given knowns; the keyword iterates is used in place of returns to indicate such a mode. For example, the contains method of the Collection class has the signature

boolean contains(Object $\mathrm{x}$ ) iterates $(\mathrm{x})$

meaning that its backward mode can be used to iterate over all contained objects. Using iterative modes, the Java collections framework could be made $35 \%$ more concise by implementing its operations, including iterators, as modes of relatively few methods [17].

\subsection{Semantics and solving}

The semantics of JMatch is defined as a syntax-directed, typepreserving translation to Java $a_{y i e l d}$ [18], which extends Java with coroutine methods in which control is yielded to the caller via the yield statement, much as in languages like C\#, Ruby, and, originally, CLU [16].

JMatch supports imperative Java code, the translation of which is relatively straightforward. The interesting parts of the translation involve the solving of boolean formulas and pattern expressions. JMatch considers a formula or pattern solvable when the compiler can generate an algorithm that either finds satisfying assignments to unknowns or determines that there are none. In the latter case, the formula or pattern is not satisfiable but is still solvable. A formula or pattern may also be satisfiable but not solvable if the compiler does not know how to generate an appropriate algorithm for determining satisfying assignments.

As an extension to Java, JMatch allows side effects, although its new features encourage a declarative programming style. With side effects, programmers need to reason about the order in which computations occur. The JMatch solver therefore solves formulas in a well-defined order that is left-to-right as much as possible.

\footnotetext{
${ }^{1}$ Note that the operator $=$ is an equality test, which unambiguously $s u b$ sumes its usual Java role as imperative assignment.
} 


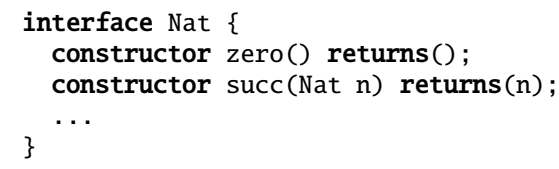

Figure 2. Natural number interface with named constructors.

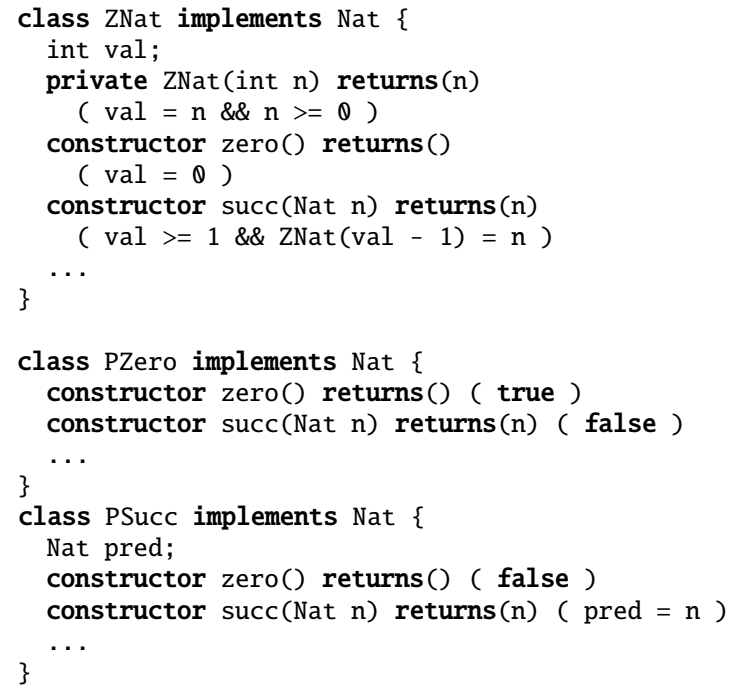

Figure 3. Three implementations of Nat.

\section{Pattern-matching extensions}

We extend JMatch, adding new pattern-matching constructs to better support object-oriented programming and data abstraction and to increase expressive power in other ways.

\subsection{Named constructors}

In JMatch, pattern matching using procedures is successful only if the value being matched is either their result or one of their arguments. Therefore, a JMatch procedure can successfully match on its own receiver object (this) only if the procedure is a constructor or happens to return its receiver object as the result. Since a constructor belongs to a particular class, code using a constructor pattern is tightly coupled to that particular implementation. This tight coupling interferes with extensibility and code reuse.

To support implementation-oblivious pattern matching, we extend JMatch with named constructors that can pattern-match an object whose run-time class is unknown. Named constructors have an explicit name different from that of their class, and they can be declared in interfaces.

For example, Figure 2 shows a Nat interface exposing two named constructors, zero and succ. Figure 3 shows two partial implementations of Nat. The first (ZNat) corresponds to the implementation of Figure 1. The second is analogous to the OCaml version and consists of two classes: PZero, representing zero, and PSucc, representing the successor of its field pred at line 18 .

Unlike ordinary constructors, a named constructor like zero can be applied to an object $x$ of type Nat or of any subtype of Nat. It acts as a boolean predicate in this style of invocation. For example, ZNat $(\theta)$.zero() evaluates to true because its implementation tests the equation val $=\mathbb{Q}$. A named constructor for type $T$ may be invoked without an explicit receiver object when it is used to pattern-match a value of type $T$. In this case, the receiver object

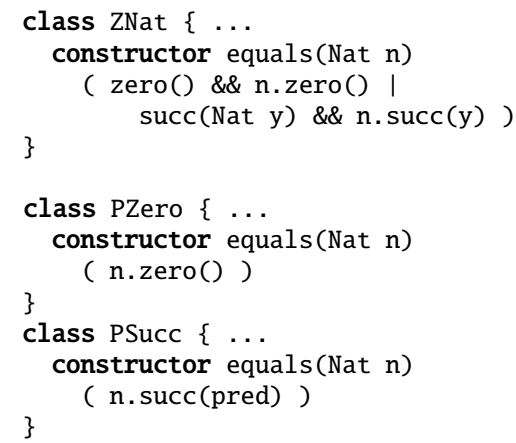

Figure 4. Equality constructors.

is the value being matched. Finally, named constructors can be invoked to construct new objects of their class, as in the expression ZNat . zero (). In the forward mode, the fields of this are in scope as unknowns to be solved for either directly in the formula or via another constructor. For example, val in the equation val $=\theta$ at line 6 is solved directly by assigning zero to it.

\subsection{Equality constructors}

As written, the implementations of Nat in Figure 3 are incomplete. The problem is that the forward mode of succ in ZNat promises to construct a ZNat from an arbitrary Nat predecessor $n$. If $n$ is not a ZNat, the equality test at line 8 between ZNat (val - 1) and $\mathrm{n}$ will fail. We fix this by adding an operation to Nat that allows solving for equality between objects of different classes:

constructor equals (Nat $n$ );

Because equals is used to pattern-match on the receiver object, it becomes a special named constructor-an equality constructorrather than an ordinary boolean method as in Java. If defined, equals is used for solving equality in addition to JMatch's default strategy of direct assignment. The code of equals for the classes implementing Nat is given in Figure 4.

Using equals, the equality ZNat (val -1 ) $=n$ is solved for non-ZNat objects $n$ by invoking ZNat . equals, defined at lines 2 4. This method tests whether $n$ is zero or the successor of some number. If the former, it returns ZNat.zero; if the latter, it invokes ZNat. succ recursively to retrieve the predecessor of $n$, which is bound to $\mathrm{y}$ by the constructor invocation $\mathrm{n} \cdot \operatorname{succ}(\mathrm{y})$. Operationally, ZNat's equals and succ interoperate to find successive predecessors until either zero or a ZNat representation (as in PSucc. succ (ZNat (3)), which is legal!) is encountered. Once equals converts $\mathrm{n}$ to a $\mathrm{ZNat}$ object, succ matches the internal representation of this ZNat object with val - 1, solving for val, which internally represents the desired successor.

\subsection{Other extensions}

A complete overview of the existing patterns in JMatch can be found in Section 2.2 of the JMatch technical report [18]. We extend the language with additional operators and a new pattern that increase expressive power:

- JMatch already has a pattern conjunction operator called as, which generalizes ML's pattern operator of the same name by requiring two arbitrary patterns to match the same value. We add a pattern disjunction operator, \#, that combines two patterns into a single pattern that matches either or both of the two, and solves for the same unknowns. For example, the formula int $\mathrm{x}=\mathrm{y}-1 \# \mathrm{y}+1$ (which should be read as int $\mathrm{x}=(\mathrm{y}-1 \#$ $\mathrm{y}+1)$ ) generates the two solutions $\mathrm{x}=\mathrm{y}-1$ and $\mathrm{x}=\mathrm{y}+1$ when 


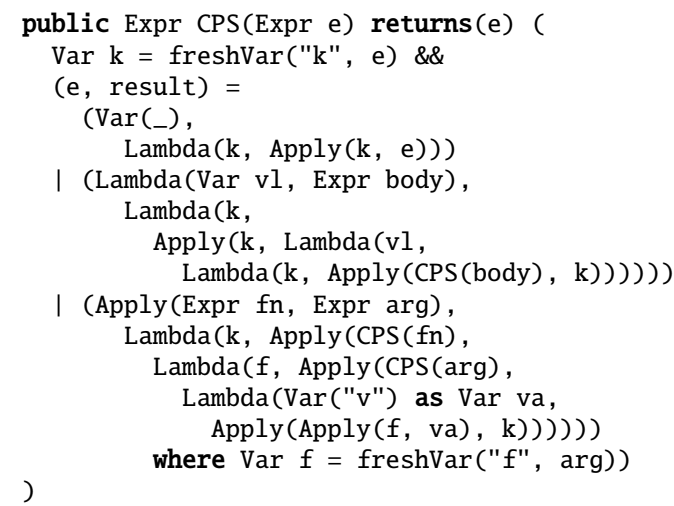

Figure 5. Invertible CPS conversion.

solving for $\mathrm{x}$, and $\mathrm{y}=\mathrm{x}+1$ and $\mathrm{y}=\mathrm{x}-1$ when solving for $\mathrm{y}$. Unlike Icon's alternation expression [9], a match is attempted against all alternatives even if one of them fails.

- We also add a disjoint disjunction operator, I, that behaves like \# except that the patterns must be disjoint. A pattern constructed with this operator produces at most one solution when a value is matched against it, unlike \#. The number of solutions is important because the pattern-matching statements require that there be only a single solution. The compiler verifies that patterns combined via $\mid$ are disjoint. The formula $x=1 \quad 2$ would therefore be legal but $\mathrm{x}=\mathrm{y}-1 \mid \mathrm{y}+1$ would not if used to solve for $\mathrm{y}$.

- A tuple pattern, written $\left(p_{1}, \ldots, p_{n}\right)$, may be used to match multiple values at once. Tuples are not first-class values; uses of tuple patterns are equivalent to, but often more concise than, a set of equations expressed over the tuple components.

These new constructs add expressiveness. For example, the JMatch 1.1.6 release [18] includes an example of invertible conversion to continuation-passing style (CPS). The same two computations, CPS conversion and its inverse, are both expressed even more concisely in Figure 5 using the new pattern operators. In this code, the use of tuples enables the translation rules to be expressed essentially as inference rules. The pattern ( $p$ where $f$ ) on line 15 refines pattern $p$ to succeed only when formula $f$ is also satisfiable. The use of $\mid$ ensures that CPS is one-to-one, though not total in its backward mode. Without I, the JMatch compiler would be unable to conclude that the three cases are disjoint and would raise the error that CPS is not one-to-one.

\section{Static annotations for exhaustiveness reasoning}

Several pattern-matching forms in JMatch can benefit from verification of exhaustiveness. As we saw in Figure 1, switch statements are one such form. Whether switch (e) $\left\{\overrightarrow{\text { case } p_{i}: s_{i}}\right\}$ is exhaustive corresponds to (roughly) whether

$$
\bigvee_{i=1}^{n} e=p_{i}
$$

is a tautology. A second such form is the JMatch statement cond $\left\{\overrightarrow{\left(f_{i}\right)\left\{s_{i}\right\}}\right\}$, which executes the first statement $s_{i}$ such that its corresponding formula $f_{i}$ is true. For exhaustiveness, at least one such formula must be true. A third pattern-matching form is let $f$, which is equivalent to cond $\{(f) \quad\{\}\}$ except that variable bindings made in $f$ are in scope for the remainder of the statement's block. The declaration int $\mathrm{x}=2$ is in fact syntactic sugar for let

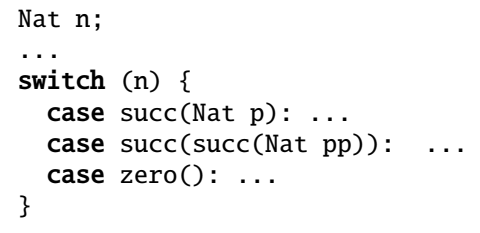

Figure 6. Redundant switch statement.

int $\mathrm{x}=2$. Therefore, the formula $f$ in a let statement should always be satisfiable.

In principle, exhaustiveness checking seems simple. Reasoning about exhaustiveness while preserving data abstraction, however, is challenging because the client code performing pattern matching is oblivious to the concrete representation (e.g., private fields) of objects. For example, given the code in Figure 6, the compiler does not know the implementation of succ and zero with which $n$ will be matched. Even if it did know, using this knowledge would violate modularity, coupling correctness of this code to implementation choices internal to Nat. Moreover, given a value of type Nat, the compiler may not assume that succ and zero are the only ways to construct the value; there could be another constructor defined in Nat that could produce the same value. As a result, the compiler does not have enough information about the patterns to show that disjunction (1) is a tautology.

To enable the compiler to reason modularly about exhaustiveness, we must expose enough information to the client about the relation implemented by a method without exposing implementation details. Supplied with this information for the code in Figure 6, the compiler should be able to determine that all values of type Nat will be matched by some case. If that were not true (e.g., if the first case were omitted), the compiler should issue a warning. Also, in the code as written, the second case is redundant because anything matching succ (succ(Nat pp)) must have matched succ(Nat p). Redundant code often indicates errors in programmer's reasoning; the compiler ought to report this too. At the same time, the exposed information should let the compiler know that zero and succ are indeed disjoint and conclude that the third case and the first two are not redundant. Without such information, the compiler could generate a false redundancy warning.

To support static verification of exhaustiveness and other properties, three new kinds of concise and intuitive specifications provide the missing information: class invariants, matches clauses, and ensures clauses. As an orthogonal benefit, all of these specifications can exploit the new pattern operator $\mid$ to prove patterns disjoint. We now explore these new features in more detail.

\subsection{Class and interface invariants}

One way to provide the information needed to determine exhaustiveness is as a class or interface invariant. For example, we can express that all instances of Nat match either zero() or $\operatorname{succ}()$ by adding the following invariant to the Nat interface, using | to assert that the two patterns are disjoint:

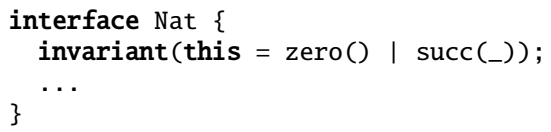

This example invariant shows how to obtain the exhaustiveness analysis provided by algebraic data types, while preserving data abstraction and allowing extensibility. New implementations of the Nat interface do not alter this invariant.

Class and interface invariants can be thought of as a kind of boolean-valued method whose value is always asserted to be true 


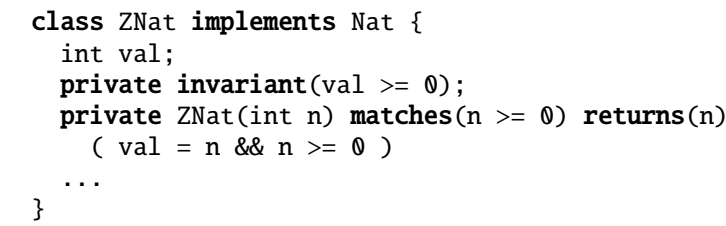

Figure 7. Private invariant and matches clause.

and whose implementation is visible to callers. Invariants may be given visibility modifiers (public, protected, or private). To maintain modularity, an invariant may only mention methods and fields that are at least as visible as the invariant itself.

Invariants not publicly visible may be useful for verifying the implementation of a class, such as the totality of the implementation of its methods. For instance, in the ZNat code of Figure 3, the field val cannot be negative. We can add a private invariant asserting this constraint, as in Figure 7. This invariant supports successful verification of the backward mode of the implementation of the constructor ZNat (), which should be total among all ZNat values. The invariant plus the first conjunct imply the second conjunct, $\mathrm{n}>=\mathbb{0}$. The private invariant also helps verify both modes of $\operatorname{succ}()$.

\subsection{Matches clauses}

One impediment to checking exhaustiveness is that a method mode may implement partial functions: on some inputs, its body might be unsatisfiable, in which case the method will fail rather than returning values for its unknowns. We extend the JMatch language with a way to specify when a method will successfully produce a result. This "matching precondition" is analogous to a precondition, but rather than specifying when a method call is legal, it specifies when pattern matching is guaranteed to succeed. The specification is conservative in that matching could succeed even when the condition does not hold.

For example, consider the constructor ZNat () in Figure 3. Any ZNat object must have a representation as a nonnegative integer. The corresponding matching precondition for the forward mode is $\mathrm{n}>=\boldsymbol{Q}$, meaning that for any nonnegative $\mathrm{n}$, there exists a ZNat object matching that $n$. This matching precondition implies the constructor body, allowing successful verification of the forward mode. The backward mode of ZNat (), on the other hand, is total, corresponding to the matching precondition true.

Asking the programmer to specify matching preconditions for each mode would be verbose and repetitive, since different modes may share knowns (i.e., inputs). Our insight is that the programmer can write a single condition that captures when matching will succeed for the entire relation implemented by a method. We call this condition the matches clause for the method. Methods having no matches clause defaults to matches(false), meaning that matching is not guaranteed to succeed for any input. The JMatch 2.0 compiler must extract the matching precondition for each mode from the consolidated matches clause. The extraction is described formally in Section 4.3; the rest of this section illustrates how extraction works for ZNat ().

The matches clause for ZNat () is shown in Figure 7. Figure 8 (a) shows the actual relation implemented by ZNat; Figure 8(b) shows the matches clause, describing the relation consisting of integral points in the shaded region. This relation can be viewed as an approximation to the true ZNat relation. Informally, the extraction obtains the matching precondition by projecting this relation onto the axis corresponding to an appropriate mode, obtaining matching preconditions shown as thick arrows. For the forward mode (returns (result)), the relation is protected onto the

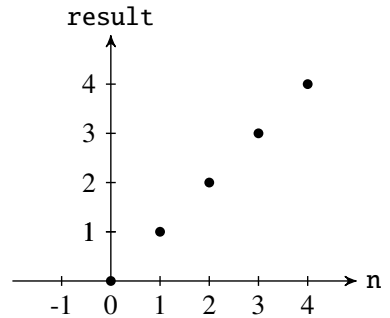

(a) actual relation

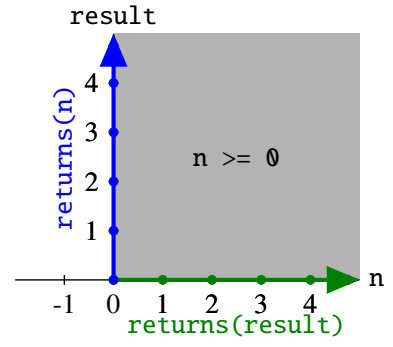

(b) matching preconditions
Figure 8. The ZNat relation.

$\mathrm{n}$ axis, obtaining $\mathrm{n}>=0$. For the backward mode (returns $(\mathrm{n})$ ), it is projected onto the result axis, obtaining true.

\subsection{Extracting matching precondition from matches clause}

In general, the body of a method implements some relation $B$ and the matches clause specifies another relation $M$. Suppose that the method is a relation over a set of variables $\{\vec{x}\}$. For each mode $\mathcal{M}$ of the method, this set is partitioned into disjoint sets of knowns (inputs) $\{\vec{k}\}$ and unknowns (outputs) $\{\vec{u}\}$. We can then view the relations $M$ and $B$ as predicates over knowns and unknowns, $M(\vec{k}, \vec{u})$ and $B(\vec{k}, \vec{u})$, respectively. Given $\vec{k}$, the precise condition in which the body guarantees success is therefore $\exists \vec{u} . B(\vec{k}, \vec{u})$. We call this formula the precise matching precondition.

For brevity, we define a function $\pi_{\mathcal{M}}$ that constructs the precise matching precondition for mode $\mathcal{M}$ by projecting an arbitary predicate $B$ onto the knowns:

$$
\left(\pi_{\mathcal{M}} B\right)(\vec{k}) \stackrel{\Delta}{\Longleftrightarrow} \exists \vec{u} . B(\vec{k}, \vec{u})
$$

Given $\mathcal{M}$ and $B, \pi_{\mathcal{M}} B$ is a predicate on $\vec{k}$ that holds when $\vec{k}$ provides some way to satisfy $B$ in mode $\mathcal{M}$ and hence to successfully pattern-match.

To preserve abstraction, reasoning about exhaustiveness must be done using the matches clause $M$, not $B$. Intuitively, if $M \Rightarrow B$, the body will be satisfiable whenever the matches clause holds; however, to require this implication would be unnecessarily restrictive. In the case of ZNat relation, for example, $\mathrm{n}>=0$ does not imply the actual relation, and the only matches clause that does so while preserving abstraction is false. A more useful correctness condition is $M \Rightarrow \wedge_{\mathcal{M}^{\prime}} \pi_{\mathcal{M}^{\prime}} B$, where $\mathcal{M}^{\prime}$ is ranges over declared modes. In other words, satisfiability of the matches clause only needs to imply satisfiability of the body in all the modes actually available. This can be visualized as expanding $B$ (in the case of ZNat, the dots in Figure 8(a)) along all the dimensions corresponding to the supported modes (obtaining, in this case, the shaded area in Figure 8(b)).

It is easy to show that the formula $M \Rightarrow \wedge_{\mathcal{M}^{\prime}} \pi_{\mathcal{M}^{\prime}} B$ is equivalent to $\pi_{\mathcal{M}} M \Rightarrow \pi_{\mathcal{M}} B$ for each declared mode $\mathcal{M}$. This suggests that given the mode $\mathcal{M}=(\{\vec{k}\},\{\vec{u}\})$ and the inputs $\vec{k}$, we should verify exhaustiveness by using $\left(\pi_{\mathcal{M}} M\right)(\vec{k})$ as the matching precondition. Unfortunately, the existential quantifiers in this formula make it illsuited to automated reasoning. Instead, we construct a weakening of $\pi_{\mathcal{M}} M$ that does not mention existential quantifiers. Let us denote this weakened predicate on $\vec{k}$ as $\operatorname{Extract}_{\mathcal{M}} M$.

The construction of Extract $_{\mathcal{M}} M$ proceeds as follows. We first convert the matches clause into negation normal form (NNF) so that the formula uses only positive logical operators over atomic formulas. We then use a variant of the usual JMatch algorithm for generating solutions to a formula. The first step is to reorder the atoms so that as many unknowns as possible can be solved from left 
to right. After this reordering, atoms that do not mention unknowns are left unchanged, as are atoms in which all unknowns are solvable in the left-to-right order. Atoms mentioning any unsolvable unknowns are dropped; that is, they are replaced with true. Any remaining occurrences of unknowns can be thought of as existentially quantified, but because each remaining unknown is solvable, it represents a solution expressed entirely in terms of knowns.

For instance, in the ZNat example, the atomic formula $n>=0$ is dropped in the backward mode because $n$ is unsolvable, leaving only true. As another example, consider extracting a matching precondition for $\mathrm{x}>0 \& \& \mathrm{y}>=0 \& \& \mathrm{x}+1=\mathrm{y}$, where $\mathrm{x}$ is unknown and $y$ known. The formula is first reordered to allow solving for $\mathrm{x}$, yielding $\mathrm{y}>=0 \& \& \mathrm{x}+1=\mathrm{y} \& \& \mathrm{x}>0$. The first atom is left unchanged because it only mentions $y$. The second is also kept because it solves fore $\mathrm{x}$, allowing the third atom to be retained as well. Because $\mathrm{x}$ is solved by the value $\mathrm{y}-1$, the extracted precondition is $y>=0 \& \&(y-1)+1=y \& \&(y-1)>0$, which is equivalent to $y>1$. In general, $x$ might be solved by a user-defined method. Section 5 explains how atoms containing such unknowns are handled.

Dropping unsolvable atoms is a heuristic, but it seems effective because such atoms are typically satisfiable for all possible values of the knowns. In general, however, dropped atoms might not be satisfiable, in which case $\operatorname{Extract}_{\mathcal{M}} M$ may not be conservative. For example, if the matches clause were instead $\mathrm{y}>=0 \& \& \mathrm{x}<\mathrm{y} \& \& \mathrm{x}>0$, dropping the atoms $\mathrm{x}<\mathrm{y}$ and $\mathrm{x}>\boldsymbol{\theta}$ would result in the extracted precondition $\mathrm{y}>=\theta$. The precise matching precondition $\left(\pi_{\mathcal{M}} M\right)(\mathrm{y})$ is rather $\mathrm{y}>=2$, since there is no satisfying assignment for $\mathrm{x}$ when $\mathrm{y}<2$.

Extract $_{\mathcal{M}} M$ can be used not only for analyzing exhaustiveness, but also for verifying that the method body implements its extracted precondition in mode $\mathcal{M}$. That is, when the method body is implemented as a formula, the compiler verifies in each mode $\mathcal{M}$ that for all inputs $\vec{k},\left(\right.$ Extract $\left._{\mathcal{M}} M\right)(\vec{k}) \Rightarrow\left(\pi_{\mathcal{M}} B\right)(\vec{k})$. This ensures soundness for exhaustiveness analysis done using $\operatorname{Extract}_{\mathcal{M}} M$. Verification is done using an SMT solver, as described in Section 6. For imperative method implementations, this verification is left to the programmer, though existing program logics might be used to obtain a verifiable logical interpretation in many cases.

\subsection{Opaquely refining matches}

In general, we may want to support modes in which precondition extraction fails because the matches clause does not or cannot capture the relationship among the arguments. For example, consider adding to ZNat () a predicate mode returns (), in which there are no unknowns. In this mode, the matches clause $n>=0$ does not correctly capture the matching precondition, yet the existing implementation is correct. To support such modes, matches clauses may be refined using the special opaque predicate notall. During precondition extraction, an atom notall $\left(\vec{x}_{i}\right)$ is treated as unsolvable if any of the variables $x_{i}$ is unknown, and is therefore dropped; if all of the variables are known or already solved, however, the predicate is treated as false.

Thus, to support a predicate mode for ZNat (), the predicate notall (result, $\mathrm{n}$ ) is conjoined with $\mathrm{n}>=0$ to indicate that pattern matching is not guaranteed to succeed when both result and $n$ are known. This notall predicate corresponds to the refinement that converts the gray area in Figure 8(b) into just the black dots in Figure 8(a). The opaque notall is needed because this refinement cannot be characterized abstractly.

\subsection{Ensures clauses}

Matches clauses are a kind of multimodal precondition. To improve the precision of verification and exhaustiveness reasoning in JMatch, we add the ensures clause, a multimodal postcondition whose syntax resembles previous, unimodal postcondition specifications (e.g., [25, 27]). The ensures clause for a method is an abstraction of the relation implemented by the method, expressed in terms that client code can understand; that is, it only mentions names a legal caller could name, similar to the specifications proposed by Leavens and Müller [14].

Unlike the matches clause, the ensures clause must define an overapproximation (a superset) of the implemented relation. Thus, in any context where a method call is known to have succeeded, the ensures clause can be assumed to hold with respect to the values supplied as knowns and the values returned as unknowns.

Because the clauses for both matches and ensures are often identical, the syntax matches ensures $(f)$ may be used as a shorthand for matches $(f)$ ensures $(f)$.

\section{Checking exhaustiveness and totality}

JMatch 2.0 must show the exhaustiveness of various patternmatching statements (switch, cond, and let). Similar verification is required for methods with a matches or ensures clause, since they promise to succeed in each mode when the extracted matching precondition is true, and since the postcondition must hold if the methods succeed. In addition, both arms of I must be verified as disjoint. In each case, the analysis constructs quantifier-free formulas that can be satisfied only if some cases are not handled by the appropriate patterns or formulas.

Section 4 described verification informally while pretending that formulas can be verified directly, e.g., by an SMT solver. This is not true in general, because formulas may contain user-defined predicates that must be treated abstractly.

To aid in constructing formulas to be verified the SMT solver, we introduce an intermediate representation language $\mathbb{F}$ that is similar to the language of quantifier-free logical formulas. Each formula is transformed to an $\mathbb{F}$ formula using function $V_{\mathcal{F}}$ defined inductively on the syntax of formulas. $\mathcal{V}_{\mathcal{F}}$ takes a formula $f$ to be transformed, along with the set $U$ of unknowns to be solved in $f$, and an additional $\mathbb{F}$ formula $F$ that represents the rest of the constraint. $\mathcal{V}_{\mathcal{F}} \llbracket f \rrbracket U F$ holds if (1) $f$ is satisfiable and (2) $F$ also holds under any solution to the unknowns in $U$ satisfying $f$.

Because space is limited, the definitions of the translation are elided here; they are available in the technical report [11].

The Z3 theorem prover [3] is used to find a model satisfying these $\mathbb{F}$ formulas. This model can be used to construct a counterexample to explain the failure of exhaustiveness or totality to the user. The verification done by $\mathrm{Z} 3$ does not affect the dynamic semantics of JMatch 2.0; it only affects warnings given to the programmer.

In the remainder of this section, let $\mu f$ be the set of variables declared in $f$.

\subsection{Verifying exhaustiveness}

Every switch and if statement can be written as a cond statement. Thus, verification of switch and if reduces to that of cond.

To verify a cond statement cond $\left\{\overrightarrow{\left(f_{i}\right)\left\{s_{i}\right\}}\right.$ else $s$, we begin by asserting the invariants of all the known variables in the context. We then proceed case by case. The algorithm first checks whether $f_{i}$ yields a solution to its unknowns. If not, the compiler issues a warning that this arm is redundant. In either case, the assertion is updated to rule out patterns matched up to the current arm. The else arm, if present, is equivalent to true.

Finally, the cond statement is exhaustive if the final assertion is unsatisfiable. If not, a counterexample is generated from a satisfying assignment, and a nonexhaustive warning is reported.

A cond statement can be used to refine patterns in the same way as a where pattern. Since both switch and if are syntactic sugar for cond, so can they. Let $I$ be the invariant prior to the 
verification of a conditional case $(f)\{s\}$. We verify $s$ with the stronger invariant $I^{\prime} \triangleq I \wedge\left(\mathcal{V}_{\mathcal{F}} \llbracket f \rrbracket \mu f\right.$ true $)$.

To verify let $f$, we check whether the negation of $f$ is satisfiable. If so, a warning that the let statement may not always be total is reported to the programmer.

\subsection{Verifying matching specifications}

As described in Section 4.3, the bodies of methods are checked against the matches clause of the method to ensure that the body succeeds whenever the matches clause is true. Recall that this entails verifying the proposition Extract $_{\mathcal{M}} M \Rightarrow \pi_{\mathcal{M}} B$.

One complication is that the matches clause $M$ of a method may refer to other methods. These method references may solve for unknown variables in $M$. In turn, these unknowns may be further referenced by other atoms in $M$, imposing additional matching preconditions.

The matches and ensures clauses of the referenced methods are used to resolve this complication. The matches clause imposes additional matching precondition to $M$, and the ensures clause constrains the values of unknowns that may be referenced later in $M$.

In the following example, the matches clause of method bar refers to foo:

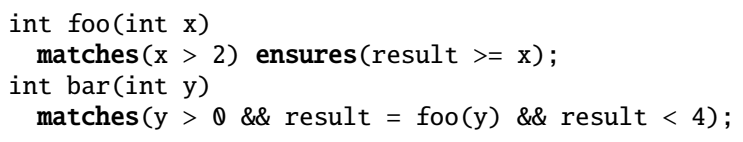

Now, suppose we want to extract bar's matching precondition for the forward mode, i.e., when y is known. The reordering and atomdropping procedure does not alter the matches clause. This means $\operatorname{bar}(\mathrm{y})$ succeeds if $\mathrm{y}>\boldsymbol{\theta}$, foo(y) returns a result, and foo(y) $<4$. The invocation of foo in bar's matches clause succeeds if $y>2$, and foo's ensures clause says result $\geq \mathrm{y}$. Therefore, $\operatorname{bar}(y)$ is guaranteed to succeed if $y>0 \wedge y>2 \wedge$ result $\geq$ $\mathrm{y} \wedge$ result $<4$, which is equivalent to $\mathrm{y}=3$.

We now give the formal translation for $\operatorname{Extract}_{\mathcal{M}} M$, where $\mathcal{M}=$ $(\{\vec{k}\},\{\vec{u}\})$. If $\hat{M}$ is the result of reordering and dropping atoms in $M$, and $\{\overrightarrow{\hat{u}}\} \subseteq\{\vec{u}\}$ is the set of unknowns remaining in $\hat{M}$, then we have

$$
\text { Extract }_{\mathcal{M}} M \triangleq \mathcal{V}_{\mathcal{F}} \llbracket \hat{M} \rrbracket(\{\overrightarrow{\hat{u}}\} \cup \mu \hat{M}) \text { true }
$$

Similarly, the precise matching precondition is defined as

$$
\pi_{\mathcal{M}} B \triangleq \mathcal{V}_{\mathcal{F}} \llbracket B \rrbracket(\{\vec{u}\} \cup \mu B) \text { true }
$$

With the above definitions, we are ready to formally define the verification conditions for JMatch methods. To verify a method

$$
T_{r} \text { foo }\left(\overrightarrow{T_{i} x_{i}}\right) \text { matches }(M) \text { ensures }(E)
$$

in mode $\mathcal{M}$ with body $B$, we prove these two assertions:

$$
\begin{aligned}
\text { Extract }_{\mathcal{M}} M & \Rightarrow \pi_{\mathcal{M}} B \\
\pi_{\mathcal{M}} B & \Rightarrow \mathcal{V}_{\mathcal{F}} \llbracket E \rrbracket \mu E \text { true }
\end{aligned}
$$

Assertion (2) says that if the extracted matching precondition for $\mathcal{M}$ holds, then $B$ succeeds in generating a solution to all of its unknowns, which can be part of the arguments or declared in $B$ itself. Assertion (3) says that if $B$ succeeds in generating a solution, then the postcondition of the method holds.

For a method declared in an interface or declared abstract, and for each mode $\mathcal{M}$ declared in that method, the assertion

$$
\text { Extract }_{\mathcal{M}} M \Rightarrow \text { Extract }_{\mathcal{M}} E
$$

is proven instead. Since the matches clause must specify an underapproximation of the (unimplemented) relation and the ensures clause an overapproximation, this assertion says that by transitivity, if the matching precondition holds, then the postcondition should hold as well.

Failure to prove these assertions means that the method does not respect its contract. As a result, a warning for the violation is issued to the programmer.

\subsection{Verifying disjoint patterns}

JMatch 2.0 verifies multiplicity of formulas and patterns, ensuring that they generate at most one solution in non-iterative modes. Disjoint pattern disjunctions allow disjunctions to be expressed without generating multiple solutions, but this property must be verified. We also overload the | symbol as a logical operator; the formula $f_{1} \quad \mid f_{2}$ is a disjunction that may be used only if at most one of $f_{1}$ or $f_{2}$ is satisfiable. Let $U$ be the set of unsolved unknowns in $p_{1} \mid p_{2}$, and let $p_{1}^{\prime}$ be the result of substituting each unsolved unknown in $p_{1}$ with a fresh variable, and similarly for $p_{2}^{\prime}$. Patterns $p_{1}$ and $p_{2}$ are disjoint if $\left(\mathcal{V}_{\mathcal{F}} \llbracket x=p_{1}^{\prime} \rrbracket U\right.$ true $) \wedge\left(\mathcal{V}_{\mathcal{F}} \llbracket x=\right.$ $p_{2}^{\prime} \rrbracket U$ true), where $x$ is a fresh variable, is unsatisfiable. Similiarly, when $\mid$ is used as a logical operator, formulas $f_{1}$ and $f_{2}$ are disjoint if $\left(\mathcal{V}_{\mathcal{F}} \llbracket f_{1}^{\prime} \rrbracket U\right.$ true $) \wedge\left(\mathcal{V}_{\mathcal{F}} \llbracket f_{2}^{\prime} \rrbracket U\right.$ true $)$ is unsatisfiable.

Consider the examples in Section 3.3. The pattern $1 \mid 2$ is disjoint because $x=1 \wedge x=2$ is unsatisfiable. The disjunction $y-1 \mid y+1$ is disjoint when $\mathrm{y}$ is known. When y is unknown, the verification procedure renames $\mathrm{y}$ in each arm to a fresh variable, yielding $x=\mathrm{y}_{1}-1 \wedge x=\mathrm{y}_{2}+1$, which is satisfiable, so the compiler generates a warning.

\subsection{Soundness}

As in most functional programming languages, we consider failures of exhaustiveness not as errors but rather as a reason to warn the programmer. Our goal is to help programmers be effective. Therefore, some unsoundness or incompleteness may be tolerable or even desirable if it rarely limits or annoys the programmer. Our verification procedures establish two main sources of unsoundness, possibly leading to erroneous warnings or lack of warnings. An obvious source is that JMatch is an imperative language, yet the reasoning procedures described here do not take side effects into account. We do not consider this a serious problem because JMatch encourages a programming style in which side effects are used sparingly and are encapsulated inside data abstractions. A second source of unsoundness arises from recursively defined methods, which are discussed in Section 6.2. In some cases, the compiler may report that it cannot prove exhaustiveness or lack of redundancy. This does not seem to be a problem in practice.

\section{Implementation}

We have built a prototype implementation of JMatch 2.0 by extending the JMatch 1.1.6 compiler [18] to add the new pattern matching features in Section 3 and the static annotations in Section 4, and to use the Z3 theorem prover [3] to verify exhaustiveness, totality, and multiplicity.

\subsection{Translating new features}

Each named constructor foo (..) defined in class $C$ is translated into two JMatch methods having the same visibility as that of foo. The first method is boolean foo (...) and contains all the modes where result is known. The other method is static C create $\$ f o o(.$.$) ) and contains the remaining modes, whose$ body requires creating a fresh object. For named constructors defined in an interface, the latter translation is omitted. An invocation of a named constructor is also transformed to use one of the translated methods accordingly, with the exception of invocations appearing in invariants and matches and ensures clauses. These invocations are retained as a type object of the class and will be 


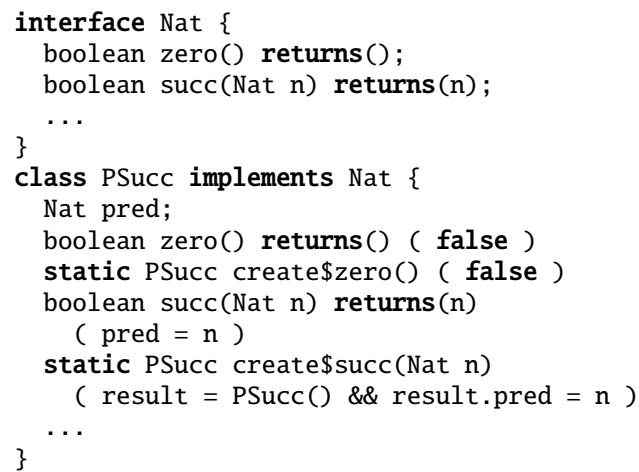

Figure 9. Translation of named constructors.

used directly during verifications. An example translation of Nat and PSucc is shown in Figure 9.

In the JMatch implementation, when a variable $w$ of type $T_{w}$ is matched against a value $x$ of type $T_{x}$, only an instanceof check is introduced if $T_{w}$ is not a supertype of $T_{x}$. To use the equality constructor, JMatch 2.0 further checks whether an equality constructor accepting one argument of type $T_{x}$ exists in the implementation of $T_{w}$ and invokes it on $x$ if the instanceof check fails.

\subsection{Handling recursion}

The verification functions defined in Section 5 unwind all method invocations appearing in a formula being translated into assertions expressed in terms of the matches and ensures clauses of the methods. In general, these translations may not be well-founded when the matches and ensures clauses of methods are mutually dependent, or in invariants of mutually recursive types. Nevertheless, the verification may be successful without fully unrolling all facts about method calls and types. We use Z3's external theory plugin to implement lazy assertions by introducing interpreted theory predicates and functions. Our external theory for Z3 expands facts about type invariants and about matching preconditions and postconditions only when instances of the theory predicates are assigned a truth value. For example, if an instance of the predicate on procedure invocation is assigned false, the negation of the matches clause of the associated procedure is asserted on the procedure inputs. If the instance is assigned true, the ensures clause is asserted. An interpreted theory function is used to enforce the uniqueness of procedure outputs when the procedure is a (partial) function.

Because Z3 treats each asserted axiom as global, every instantiated axiom is asserted as an implication whose premise is the assigned predicate. Z3 also keeps track of every asserted theory predicate in its logical context, which allows proving exhaustiveness using class invariants without unrolling them entirely. To prevent unbounded unrolling, iterative deepening [12] is used to unroll as deeply as possible within a time budget. Since the theory will not further expand facts beyond the maximum depth, Z3 concludes that no satisfying assignment exists. If this happens when checking exhaustiveness, the compiler warns that it did not find a counterexample to exhaustiveness, but that there might be one.

\section{Evaluation}

Our evaluation of JMatch 2.0 aims to answer three kinds of questions:

- Is the extended language expressive? In particular, does it permit concise implementations? What annotation burden is incurred by programmers using the new verification procedures?

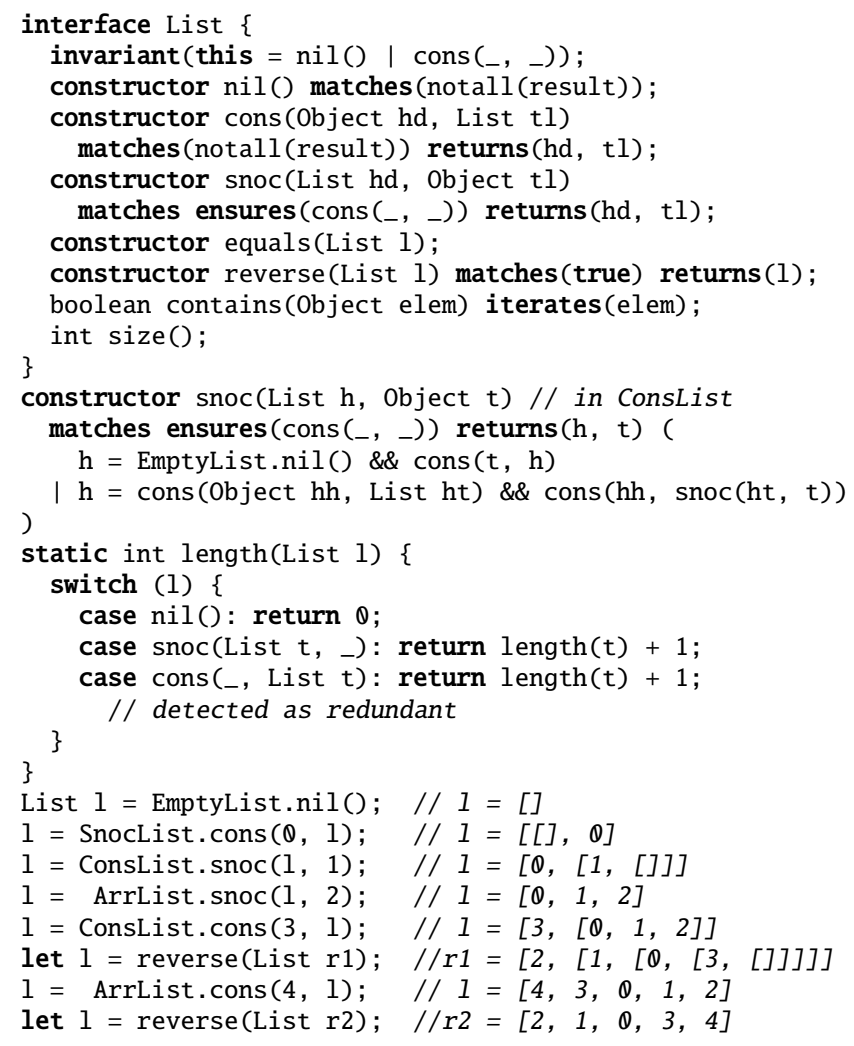

Figure 10. List interface and sample usage.

- Is the verification performed by our implementation effective on different kinds of code?

- What is the compile-time overhead of verification?

\subsection{Code examples}

We have evaluated our prototype JMatch 2.0 implementation on a variety of different coding problems. For each of these code examples, we have shown that the compiler correctly performs the three verification tasks described above, and we have measured the time taken by verification and compared it to total compilation time. To evaluate expressiveness, we have also implemented each example as concisely as we could using Java.

Natural numbers The implementations of natural numbers shown earlier in the paper are also used for our evaluation.

Lists A JMatch 2.0 interface List for immutable lists is shown in Figure 10. We implement this interface in four very different ways: the empty list (EmptyList), regular cons lists (ConsList), snoc lists (SnocList) in which elements are appended to the end, and lists with an array representation (ArrList), in which the underlying array is imperatively updated by cons but is shared as much as possible across multiple lists that record indices into it. To give the flavor of these implementations, the figure shows how the multimodal named constructor snoc is defined for ConsList. As the remaining code in the figure shows, these four list implementations interoperate smoothly, and list operations, even including reverse, can be used as patterns.

CPS We implement CPS conversion of a simple abstract syntax tree (AST) for lambda calculus; though Figure 5 shows only the key code, the implementation also includes AST classes. 


\begin{tabular}{r||c|c||c|c}
\multicolumn{1}{r|}{ Implementation } & JMatch & Java & w/o verif & w/ verif \\
\hline \hline Nat & $41(21)$ & 29 & 0.100 & 0.104 \\
PZero & 85 & 189 & 0.258 & 0.331 \\
PSucc & 98 & 226 & 0.280 & 0.435 \\
ZNat & 161 & 319 & 0.377 & 0.459 \\
\hline List & $114(72)$ & 91 & 0.129 & 0.123 \\
EmptyList & 164 & 455 & 0.416 & 0.510 \\
ConsList & 309 & 1007 & 0.807 & 2.47 \\
SnocList & 311 & 1006 & 1.05 & 3.36 \\
ArrList & 473 & 1208 & 0.864 & 1.90 \\
\hline Expr & $96(57)$ & 80 & 0.710 & 0.846 \\
Variable & 192 & 434 & 0.689 & 0.852 \\
Lambda & 239 & 500 & 1.20 & 1.52 \\
TypedLambda & 86 & 92 & 1.38 & 1.57 \\
Apply & 232 & 506 & 1.15 & 2.31 \\
CPS & 325 & 1279 & 7.88 & 8.37 \\
\hline Type & 154 & 187 & 0.218 & 0.307 \\
BaseType & 73 & 163 & 0.350 & 0.443 \\
ArrowType & 82 & 189 & 0.357 & 0.444 \\
UnknownType & 154 & 245 & 0.372 & 0.490 \\
Environment & 211 & 310 & 0.695 & 0.862 \\
\hline Tree & $114(44)$ & 69 & 0.165 & 0.170 \\
Leaf & 124 & 351 & 0.420 & 0.510 \\
Branch & 202 & 553 & 0.529 & 0.682 \\
AVLTree & 439 & 720 & 2.84 & 9.01 \\
\hline ArrayList & 773 & $1098^{*}$ & 1.67 & 1.81 \\
LinkedList & 886 & $1232^{*}$ & 2.00 & 2.20 \\
HashMap & 1082 & $1874^{*}$ & 3.41 & 3.66 \\
TreeMap & 3606 & $3955^{*}$ & 5.90 & 6.43 \\
& & & &
\end{tabular}

Table 1. The number of tokens for implementations in JMatch 2.0 versus Java. Interface token counts are reported both with and without (in parentheses) matches and ensures clauses. Verification overhead is given in seconds as the average of 24 runs, with a standard deviation of at most $15 \%$. Some comparisons (*) are versus a PolyJ [21] implementation that is more concise than the Java one. For example, the PolyJ TreeMap is $20 \%$ shorter than the Java equivalent [17].

Type inference We implement unification-based type inference over the same ASTs, augmented with type declarations. The code for type inference is placed within the AST node classes.

Trees A JMatch 2.0 interface Tree for binary trees is shown in Figure 11. We implement the AVL tree based on this interface. The rebalance method, also shown in the figure, returns the balanced version of the input subtree having $\mathrm{v}$ as the value at the root and 1 and $r$ as its children. The invariant of Tree and the ensures clause of branch are crucial for the JMatch 2.0 compiler to verify that the formula in rebalance covers all the possible input subtrees. Checking the disjoint disjunctions also ensures that there is only one way to match each tree.

Collections We convert the prior JMatch reimplementation of the key collection classes from the Java collections framework [17] into JMatch 2.0. This code base includes implementations of various data structures: hash tables, red-black trees, and resizable arrays.

\subsection{Expressiveness}

We can assess the expressiveness of JMatch 2.0 by comparing the number of language tokens needed to implement each of the examples. The resulting token counts shown in Table 1 indicate that JMatch 2.0 code is considerably more concise than in Java: $42.9 \%$ shorter on average. This conciseness is largely due to the JMatch support for modal abstraction and for equality constructors.

\subsection{Effectiveness}

There are three new verification tasks. First, switch and related constructs (let, cond, etc.) should be exhaustive. Second, method implementations must be correct with respect to both their declared matches clause and their ensures clause. Third, disjoint disjunctions must indeed be disjoint, to verify multiplicity.

All of the examples shown in the table, and all prior examples shown in the paper, are successfully verified for exhaustiveness, (non-)redundancy, and multiplicity. The compiler caught several subtle exhaustiveness bugs during development of this code, such as incorrect order of arguments to methods and invocation to an unexpected implementation of overloaded or overridden methods. In case of TreeMap, the absence of red-black tree invariants results in a nonexhaustive warning in the balance method.

\subsection{Efficiency}

Table 1 shows that verification time is reasonable for all of the code examples, even with our unoptimized prototype implementation. The reported numbers include compilation time of dependencies but exclude the overhead of initializing the compiler $(689 \mathrm{~ms})$ and the $\mathrm{Z} 3$ solver $(680 \mathrm{~ms})$. On average, the verification overhead on the evaluated code is $37.5 \%$ compared to the regular compilation time.

The speed of verification is not surprising, because verification is performed one method at a time. Verification is simple and tractable because the abstraction mechanisms we introduced to JMatch allow both programmers and the SMT solver to reason locally about code.

Because JMatch 2.0 does not significantly change the dynamic semantics of JMatch, the translation to Java is essentially unchanged. The performance of the compiled programs is therefore similar to one in the previous evaluation [17].

\section{Related work}

Integrating pattern matching with objects and data abstraction has been the subject of quite a few research efforts.

Case classes in the Scala programming language [22], as in Pizza [23], provide pattern matching by allowing case-class constructors in case arms. Scala uses sealed classes to limit the number of case classes that can inherit them. This makes exhaustiveness easy to verify, but sacrifices extensibility because only one implementation is allowed per declaration of a sealed class. Our invariant declaration achieves the same level of exhaustiveness checking but allows programmers to extend classes freely. Closely related approaches include extensible algebraic data types [32] and polymorphic variants [8], which support some extensibility and deep pattern matching, but tie pattern matching to the data representation more than is ideal.

Wadler's views [30] were an early, influential generalization of pattern matching. Views require an explicitly defined bijection between the abstract view and the representation. Unlike in our language, views do not reconcile pattern matching with subtyping and do not allow matching without knowing the identity of the implementation.

Extractors are introduced in [5] as an alternative to case classes that is compatible with data abstraction. Each extractor contains apply and unapply methods, called implicitly during construction and pattern matching. There is no check that these methods are inverses, however. Modal abstractions in JMatch are less verbose and reduce the chance of such errors. No exhaustiveness checking was proposed for extractors. Dotta et al. [4] verify extractors by relying on sealed classes, and support user-defined constructor patterns. Their work does check for pattern disjointness; abstraction prevents us from making this guarantee. 


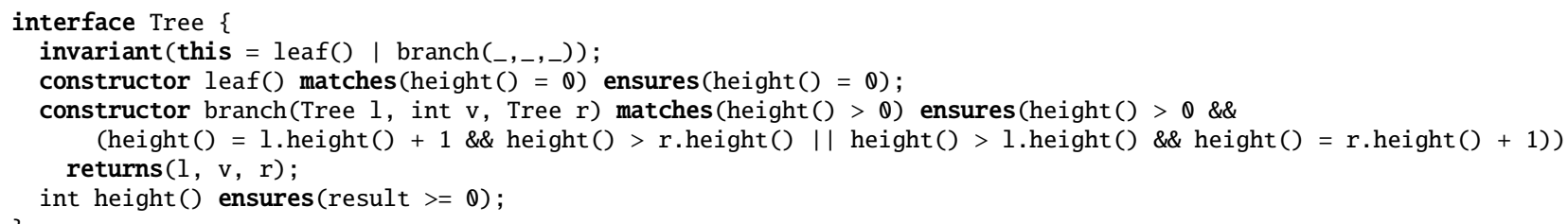

Figure 11. Tree interface and the AVL tree rebalance method, which uses the interface to check for totality.

Active patterns in F\# [29] are similar to extractors, but support exhaustiveness checking by allowing the declaration that a set of patterns is complete. Because they offer only a backward mode, they do not support algebraic reasoning in the same way as modal abstractions. They also do not support object-oriented extensibility.

The RINV language of Wang et al. [31] also uses invertible computation to implement pattern matching that is compatible with data abstraction. Rather than extracting computations from a logical characterization of the computation, RINV instead uses a restricted language for abstraction functions that guarantees invertibility. These functions are bidirectional rather than fully multimodal and do not support iterative modes. RINV analyzes exhaustiveness via specifications of complete sets of constructors, but does not verify these specifications. RINV supports neither subtyping nor extensibility.

Suter et al. [27] also use abstraction functions to reduce algebraic data types to abstract values such as multisets, and use known theories of these abstract values to reason about data types. Methods may be annotated with a postcondition in terms of abstraction functions. Leon [28] extends this reasoning to recursive programs. Used in conjunction with sealed classes, these decision procedures assist in a more precise analysis of pattern exhaustiveness by taking type refinement into account. These decision procedures do not support modal abstraction.

An orthogonal approach to integrating pattern matching into object-oriented languages is predicate dispatch $[6,20]$, which extends multimethods with the ability to choose an implementation based on general predicates over the arguments. Predicate dispatch appears to be largely orthogonal and complementary to the pattern matching mechanisms described here. The predicates in prior work on predicate dispatch are, however, less expressive than those we have explored here. OOMatch [24] uses pattern matching in predicate dispatch. Its deconstructors are similar to the backward mode of JMatch constructors. OOMatch's pattern matching differs in that it can appear only in method headers as part of predicate dispatch, and no separation of specification and implementation is provided. HydroJ [15] uses predicate dispatch to express extensible communication patterns in distributed systems; however, pattern matching is done over concrete data structures, tagged trees.

Matchete [10] extends Java with pattern matching operators similar to extractors, but matches on regular expressions and other specialized expressions. It does not analyze exhaustiveness.

The Thorn language integrates patterns to make code more concise and robust [1]. Its rich set of patterns includes boolean com- binations of patterns, general list patterns, regular expressions, and first-class patterns. First-class patterns in Thorn provide pattern abstraction that supports evolution of the data structure used in pattern matching, but Thorn does not support multiple implementations. As a dynamic language, Thorn does not check exhaustiveness.

Harmony and the Boomerang language [2, 7] support bidirectional computations over trees and strings through domain-specific lens combinators. The types in these languages support reasoning about the totality of transformations in these domains, but data abstraction is not a feature of these languages.

One focus of research on pattern matching has been on how to generate efficient code that shares computation across different patterns (e.g., [13]). Such optimizations are orthogonal to this work.

\section{Conclusions}

A clean integration of pattern matching into the object-oriented setting could simplify many programming tasks. Prior work has not managed to provide expressive pattern matching with strong data abstraction and subtyping, along with statically checked exhaustiveness. This is the first work that manages to combine these important features. We improved the integration of pattern matching with object-oriented programming, yet showed that even with this more powerful pattern matching, it is possible to reason statically about exhaustiveness, redundancy, totality, and multiplicity.

The most important insight was that programmers need to be able to specify the precondition for successful pattern matching in an abstract way. We showed that it is possible to do this while keeping the annotation burden low, by automatically extracting matching preconditions. The specification techniques introduced may be helpful for other models of multidirectional computation.

\section{Acknowledgments}

Soam Vasani and Denis Bueno got the JMatch exhaustiveness project off to a good start. Leo de Moura helped with using Z3. Ross Tate, Owen Arden, Aslan Askarov, Jed Liu, Harry Terkelsen, Mark Reitblatt, Robert Soulé, Mike George, and Danfeng Zhang offered many helpful suggestions for the presentation.

This research was supported in part by Office of Naval Research Grants N00014-09-1-0652 and N00014-13-1-0089, and by a grant administered by the Air Force Research Laboratory. 


\section{References}

[1] Bard Bloom and Martin J. Hirzel. Robust scripting via patterns. In Proc. 8th symposium on Dynamic languages (DLS), pp. 29-40, 2012.

[2] Aaron Bohannon, J. Nathan Foster, Benjamin C. Pierce, Alexandre Pilkiewicz, and Alan Schmitt. Boomerang: Resourceful lenses for string data. In Proc. 35th ACM Symposium on Principles of Programming Languages (POPL), pp. 407-419, January 2008.

[3] Leonardo de Moura and Nikolaj Bjørner. Z3: an efficient SMT solver. In Proceedings of the Theory and Practice of Software, 14th Int'l Conf. on Tools and Algorithms for the Construction and Analysis of Systems, pp. 337-340, 2008.

[4] Mirco Dotta, Philippe Suter, and Viktor Kuncak. On static analysis for expressive pattern matching. Technical report, École Polytechnique Fédérale de Lausanne, February 2008.

[5] Burak Emir, Martin Odersky, and John Williams. Matching objects with patterns. In Erik Ernst, editor, Proc. 21 st European Conf. on Object-Oriented Programming, volume 4609 of Lecture Notes in Computer Science, pp. 273-298. Springer Berlin Heidelberg, 2007.

[6] Michael Ernst, Craig Kaplan, and Craig Chambers. Predicate dispatching: A unified theory of dispatch. In Proc. 12th European Conf. on Object-Oriented Programming, pp. 186-211, 1998.

[7] J. Nathan Foster, Michael B. Greenwald, Jonathan T. Moore, Benjamin C. Pierce, and Alan Schmitt. Combinators for bidirectional tree transformations: A linguistic approach to the view-update problem. ACM Trans. Program. Lang. Syst., 29(3), May 2007.

[8] Jacques Garrigue. Typing deep pattern-matching in presence of polymorphic variants. In JSSST Workshop on Programming and Programming Languages, 2004.

[9] Ralph E. Griswold, David R. Hanson, and John T. Korb. Generators in Icon. ACM Transaction on Programming Languages and Systems, 3(2):144-161, April 1981.

[10] Martin Hirzel, Nathaniel Nystrom, Bard Bloom, and Jan Vitek. Matchete: Paths through the pattern matching jungle. In Proc. 10th Int'l Conf. on Practical Aspects of Declarative Languages, pp. 150-166, 2008.

[11] Chinawat Isradisaikul and Andrew C. Myers. Reconciling exhaustive pattern matching with objects. Technical report, Computing and Information Science, Cornell University, March 2013. http: //hdl.handle. net/1813/33123.

[12] Richard E. Korf. Depth-first iterative-deepening: an optimal admissible tree search. Artificial Intelligence, 27(1):97-109, September 1985.

[13] Fabrice Le Fessant and Luc Maranget. Optimizing pattern matching. In Proc. 6th ACM SIGPLAN Int'l Conf. on Functional Programming, pp. 26-37, 2001.

[14] Gary T. Leavens and Peter Müller. Information hiding and visibility in interface specifications. In Proc. 33rd Int'l Conf. on Software Engineering (ICSE), pp. 385-395, 2007.

[15] Keunwoo Lee, Anthony LaMarca, and Craig Chambers. HydroJ: object-oriented pattern matching for evolvable distributed systems. In Proc. 18th ACM SIGPLAN Conf. on Object-Oriented Programming, Systems, Languages and Applications, pp. 205-223, 2003.

[16] Barbara Liskov, Russell Atkinson, Toby Bloom, J. Eliot Moss, J. Craig Schaffert, Robert Scheifler, and Alan Snyder. CLU Reference Manual. Springer-Verlag, 1984. Also published as Lecture Notes in Computer Science 114, G. Goos and J. Hartmanis, Eds., Springer-Verlag, 1981.
[17] Jed Liu, Aaron Kimball, and Andrew C. Myers. Interruptible iterators. In Proc. 33rd ACM Symposium on Principles of Programming Languages (POPL), pp. 283-294, January 2006.

[18] Jed Liu and Andrew C. Myers. JMatch: Java plus pattern matching. Technical Report TR2002-1878, Computer Science Department, Cornell University, October 2002. Software release at http://www.cs. cornell.edu/projects/jmatch.

[19] Jed Liu and Andrew C. Myers. JMatch: Iterable abstract pattern matching for Java. In Proc. 5th International Symposium on Practical Aspects of Declarative Languages, pp. 110-127, January 2003.

[20] Todd Millstein. Practical predicate dispatch. In Proc. 19th ACM SIGPLAN Conf. on Object-Oriented Programming, Systems, Languages and Applications, pp. 345-364, 2004.

[21] Andrew C. Myers, Barbara Liskov, and Nicholas Mathewson. PolyJ: Parameterized types for Java. Software release, at http://www.cs . cornell. edu/polyj, July 1998.

[22] Martin Odersky, Lex Spoon, and Bill Venners. Programming in Scala: A Comprehensive Step-by-step Guide. Artima Incorporation, USA, 1st edition, 2008.

[23] Martin Odersky and Philip Wadler. Pizza into Java: translating theory into practice. In Proc. 24th ACM Symposium on Principles of Programming Languages (POPL), pp. 146-159, 1997.

[24] Adam Richard and Ondřej Lhoták. OOMatch: pattern matching as dispatch in Java. In Companion 22nd ACM SIGPLAN Conf. on ObjectOriented Programming, Systems, Languages and Applications, pp. 771-772, 2007.

[25] Clyde Ruby and Gary T. Leavens. Safely creating correct subclasses without seeing superclass code. In Proc. 15th ACM SIGPLAN Conf. on Object-Oriented Programming, Systems, Languages and Applications, pp. 208-228, 2000.

[26] Zoltan Somogyi, Fergus Henderson, and Thomas Conway. The execution algorithm of Mercury, an efficient purely declarative logic programming language. The Journal of Logic Programming, 29(1-3):1764, October-December 1996.

[27] Philippe Suter, Mirco Dotta, and Viktor Kuncak. Decision procedures for algebraic data types with abstractions. In Proc. 37th ACM Symposium on Principles of Programming Languages (POPL), pp. 199-210, 2010.

[28] Philippe Suter, Ali Sinan Köksal, and Viktor Kuncak. Satisfiability modulo recursive programs. In Proc. 18th Int'l Conf. on Static Analysis, pp. 298-315, 2011.

[29] Don Syme, Gregory Neverov, and James Margetson. Extensible pattern matching via a lightweight language extension. In Proc. 12th ACM SIGPLAN Int'l Conf. on Functional Programming, pp. 29-40, 2007.

[30] Philip Wadler. Views: a way for pattern matching to cohabit with data abstraction. In Proc. 14th ACM Symposium on Principles of Programming Languages (POPL), pp. 307-313, 1987.

[31] Meng Wang, Jeremy Gibbons, Kazutaka Matsuda, and Zhenjiang Hu. Refactoring pattern matching. Science of Computer Programming, in press. Available online 24 August 2012.

[32] Matthias Zenger and Martin Odersky. Extensible algebraic datatypes with defaults. In Proc. 6th ACM SIGPLAN Int'l Conf. on Functional Programming, pp. 241-252, September 2001. 